\title{
The relation between infant freezing and the development of internalizing symptoms in adolescence: A prospective longitudinal study
}

\author{
Hannah C. M. Niermann ${ }^{1,2}$ (D) | Anna Tyborowska ${ }^{1,2}$ (D) | Antonius H. N. Cillessen ${ }^{1} \mid$ \\ Marjolein M. van Donkelaar ${ }^{2,3}$ ～Femke Lammertink ${ }^{1}$ | Megan R. Gunnar ${ }^{4}$ | \\ Barbara Franke $e^{2,3,5}$ (i) | Bernd Figner ${ }^{1,2, *}$ | Karin Roelofs ${ }^{1,2, *}$
}

${ }^{1}$ Behavioural Science Institute, Radboud University, Nijmegen, The Netherlands

${ }^{2}$ Donders Institute for Brain, Cognition and Behaviour, Radboud University, Nijmegen, The Netherlands

${ }^{3}$ Department of Human Genetics, Radboud University Medical Center, Nijmegen, The Netherlands

${ }^{4}$ Institute of Child Development, University of Minnesota, Minneapolis, Minnesota, USA

${ }^{5}$ Department of Psychiatry, Radboud University Medical Center, Nijmegen, The Netherlands

\section{Correspondence}

Hannah C. M. Niermann, Donders Institute for Brain, Cognition and Behavior, Behavioural Science Institute, Radboud University, Nijmegen, The Netherlands.

Email: hcm.niermann@gmail.com

Funding information

HN was supported by a Research Talent Grant (406-13-022) from the Netherlands Organization for Scientific Research (NWO) and by a Christine Mohrmann grant of Radboud University (Nijmegen, Netherlands). KR was funded by a Vici grant (\#453-12-001) from NWO and a starting grant from the European Research Council (ERC StG2012 313749). Additional support was received from the European Community's Seventh Framework Programme (FP7/2007 - 2013) under grant agreement no. 602805 (Aggressotype).

The content is the sole responsibility of the authors and does not necessarily represent the official views of the funding agencies.

\begin{abstract}
Given the long-lasting detrimental effects of internalizing symptoms, there is great need for detecting early risk markers. One promising marker is freezing behavior. Whereas initial freezing reactions are essential for coping with threat, prolonged freezing has been associated with internalizing psychopathology. However, it remains unknown whether early life alterations in freezing reactions predict changes in internalizing symptoms during adolescent development. In a longitudinal study $(N=116)$, we tested prospectively whether observed freezing in infancy predicted the development of internalizing symptoms from childhood through late adolescence (until age 17). Both longer and absent infant freezing behavior during a standard challenge (robot-confrontation task) were associated with internalizing symptoms in adolescence. Specifically, absent infant freezing predicted a relative increase in internalizing symptoms consistently across development from relatively low symptom levels in childhood to relatively high levels in late adolescence. Longer infant freezing also predicted a relative increase in internalizing symptoms, but only up until early adolescence. This latter effect was moderated by peer stress and was followed by a later decrease in internalizing symptoms. The findings suggest that early deviations in defensive freezing responses signal risk for internalizing symptoms and may constitute important markers in future stress vulnerability and resilience studies.
\end{abstract}

\section{KEYWORDS}

5-HTTLPR, adolescence, defensive stress response, infancy, internalizing symptoms, longitudinal analysis 


\section{1 | INTRODUCTION}

Freezing, characterized by bodily immobility and decreased heart rate, is one of the most common defensive reactions to threatening situations, widely observed across species. This parasympathetically dominated temporary break on the motor system is known to promote selection of adequate coping reactions by facilitating perception, risk assessment, and action preparation (Blanchard, 2017; Kozlowska, Walker, McLean, \& Carrive, 2015; Roelofs, 2017). Alterations in this basic defensive stress reaction have been linked to long-lasting internalizing symptoms (i.e., signs of anxiety and depression) in animals (Kalin \& Shelton, 2003; Qi et al., 2010). Recent cross-sectional studies have confirmed the association between prolonged freezing and internalizing symptoms in humans (Kozlowska et al., 2015; Niermann et al., 2017). Although objective, early life risk markers are needed, the predictive value of early alterations in freezing for the later development of internalizing symptoms is not known. Therefore, this study examined prospectively whether and how alterations in infant freezing predict the development of internalizing symptoms from childhood into late adolescence.

The tendency to reduce activity when exposed to threat has been observed frequently in infants (Buss, Davidson, Kalin, \& Goldsmith, 2004). Given the relative helplessness of infants, this reduced activity may represent an important coping response even to mildly threatening situations. Freezing is likely generated by midbrain serotonin-mediated projections, specifically amygdala-periaqueductal gray projections to the medulla and spinal cord (Roelofs, 2017). Whereas immediate freezing in threatening situations is considered adaptive, prolonged freezing can be a sign of reduced flexibility to respond to environmental changes and has been suggested to prevent adequate stress coping (Buss \& Larson, 2000; Hagenaars, Oitzl, \& Roelofs, 2014). Indeed, animal work has shown that freezing is related to more complex approach-avoidance decision making and has been associated with subsequent decisions towards avoidance (Campese McCue, Lázaro-Muñoz, LeDoux, \& Cain, 2013). This is relevant as avoidance is the main maintaining and perhaps even causal factor of internalizing symptoms (Craske, 2003). Recent human work mainly in adults also has shown that prolonged freezing-or poor recovery from an initial freezing response-predicts subsequent instrumental avoidance responses (Ly, Huys, Stins, Roelofs, \& Cools, 2014; Ly et al., 2016) and is associated with increased internalizing symptoms (Kozlowska et al., 2015; Niermann et al., 2017). Therefore, we hypothesized that freezing is adaptive, but that alterations in this defensive response early in life-in the form of longer freezing-will predict increasing levels of internalizing symptoms in childhood to late adolescence. In addition, given emerging evidence for a proposed nonlinear association between freezing and psychopathology (Fragkaki, Roelofs, Stins, Jongedijk, \& Hagenaars, 2017), we also explored potential detrimental outcomes associated with reduced freezing behavior: Absence of or reduced freezing-in a context where freezing is a typical response in most individuals-may reflect maladaptive stress coping,

\section{Highlights}

- This prospective longitudinal study investigated whether and how deviations in infant freezing predict changes in internalizing symptoms from childhood into late adolescence.

- Deviations in infant freezing-both absent and longer freezing-were associated with relative increases in internalizing symptoms.

- Whereas absent infant freezing predicted a continuous increase from relatively low to relatively high internalizing symptoms up until late adolescence, longer infant freezing predicted increased internalizing symptoms during early adolescence only.

- Early deviations in freezing may mark an individual's risk to develop internalizing symptoms and may be important to consider in future stress vulnerability and resilience studies.

which in turn could be related to internalizing symptoms as well (Adenauer, Catani, Keil, Aichinger, \& Neuner, 2010; Fragkaki et al., 2017; Stoffels, Nijs, Spinhoven, Mesbah, \& Hagenaars, 2017).

The freezing response shows high heritability and stability in animals and humans (Niermann, Figner, Tyborowska, Cillessen, \& Roelofs, 2018), but is also sensitive to stress (Rogers, Shelton, Shelledy, Garcia, \& Kalin, 2008). Accordingly, human diathesisstress models propose that individuals' vulnerability in the form of altered freezing leads to internalizing symptoms, particularly when vulnerable individuals are exposed to stressful life circumstances (e.g., Zuckerman, 1999). Poor quality of relationships with parents and peers highly impact the developing individual (Sebastian, Viding, Williams, \& Blakemore, 2010). Therefore, our second hypothesis was that these social stress factors would further strengthen the association between altered infant freezing and the development of internalizing symptoms.

Finally, in line with the role of serotonin in freezing, genetic variation in the gene encoding the serotonin transporter (SLC6A4) has been associated with altered freezing and increased vulnerability to develop internalizing symptoms in animals (Bethea et al., 2004; Homberg, 2012). In humans, variations in SLC6A4/5-HTT/SERT have been linked to depression, especially in conjunction with stress (Bleys, Luyten, Soenens, \& Claes, 2018). We therefore explored whether individuals carrying depression-risk alleles of the functional variant in SLC6A4/5-HTT/SERT (called 5-HTTLPR) display alterations in freezing responses as infants.

We tested our hypotheses prospectively in a longitudinal study (e.g., Niermann et al., 2015; van Bakel \& Riksen-Walraven, 2002) that allowed us to relate systematic observations of freezing in 15 -month-old infants to internalizing symptoms across development into late adolescence (ages 5, 9, 12, 14, and 17). 


\section{2 | METHOD}

\section{1 | Participants}

Participants were part of the Nijmegen Longitudinal Study (NLS), which started in 1998 with a community-based sample of 129 infants at 15 months of age $\left(48 \%\right.$ females, $M_{\text {age }}=14.88$ months, $S D=0.25$, range $_{\text {age }}: 14.37-15.42$ ) recruited via health care centers in Nijmegen (the Netherlands). The children and their families were followed every 2-3 years (Table 1 ) and were representative of the Dutch population (for recruitment and family characteristics, see van Bakel and Riksen-Walraven (2002)). Participants were excluded from analyses $(n=13)$ if there was no videotape material (i.e., infant was not confronted with the situation used to assess infant freezing behavior), if infant freezing could not be reliably assessed (e.g., due to poor video quality, over-involvement of primary caregiver, infant tiredness), and/or if self- and parentreported internalizing data were lacking. Some participants had no self-reported $(n=4)$ or parent-reported $(n=1)$ internalizing data, resulting in sample sizes of 112 participants (46\% females) for the self-reported internalizing analyses and 115 participants $(46 \%$ females) for the parent-reported internalizing analyses. Genetic data on 5-HTTLPR, a functional polymorphism in the regulatory region of SLC6A4, was available for 95 of the 116 participants included in current analyses. Here, we also included information on a functional single nucleotide polymorphism in 5-HTTLPR, rs25531, resulting in the genotype-groups: (a) $L^{\prime}$-homozygotes $\left(\mathrm{L}_{A} / \mathrm{L}_{A}\right): n=27$; (b) L'/S'-heterozygotes $\left(\mathrm{L}_{A} / S, \mathrm{~L}_{A} / \mathrm{L}_{G}\right): n=47$; (c) $\mathrm{S}^{\prime}$ homozygotes $\left(\mathrm{L}_{G} / \mathrm{L}_{G}, \mathrm{~S} / \mathrm{L}_{\mathrm{G}}, \mathrm{S} / \mathrm{S}\right): n=21$ (procedures of collection and isolation of genetic material, as well as of genotyping are described in Supporting Information Appendix S1). For information on socioeconomic status and attrition analyses, see Supporting Information Appendix S2. Prior to participation, parents gave informed consent, later accompanied by children's informed assent. Participants received either a small gift or were financially reimbursed for participation. The study was approved by the local ethics committee (CMO region Arnhem-Nijmegen, the Netherlands).

\subsection{Procedures and measures}

\subsection{1 | Infant freezing}

Freezing behavior at 15 months was assessed from videotapes recorded during a robot-confrontation paradigm, which was adapted from Mullen, Snidman, and Kagan (1993) and has been previously described for the NLS by van Bakel and Riksen-Walraven (2004). During this 3-min paradigm, participants were confronted with a colorful mechanical robot (Supporting Information Appendix S3). Freezing was defined as a marked decrease in activity lasting for $\geq 3 \mathrm{~s}$ with little or no bodily movement (Buss et al., 2004) combined with no vocalization (Kalin \& Shelton, 1989). A trained coder watched all videos and coded the duration of each freezing episode using a stopwatch. Reliability was determined based on having $22 \%$ of the videotapes coded by a second trained coder, resulting in a moderateto-good intra-class correlation of $0.76,95 \% \mathrm{Cl}[0.66,0.83]$.

Total scores were created by summing the duration of an infant's freezing episodes. To control for slight variations in time of exposure to the robot, proportion scores were computed by dividing the total duration of freezing by the total duration that the infant experienced the situation $(M=0.11, S D=0.10$, range: $0.00-0.41$; see Supporting Information Appendix S3 for more descriptive information on infant freezing). Infant freezing showed only weak to moderate associations with other temperamental fearfulness measures, suggesting that freezing can be considered as a partially separate construct (see Supporting Information Appendix S3 and Table S1). ${ }^{1}$

\subsection{2 | Self-reported internalizing symptoms}

Because self-reflection on internalizing symptoms may change with development, age-appropriate measures of anxiety and depression were selected to reliably assess internalizing symptoms at ages 9, 12, 14, and 17 (Table 1; see Supporting Information Appendix S5 for justification, validity, and reliability of the measures). To arrive at one score for internalizing symptoms per age and to deal with the missing values at the item and scale levels, we computed compound scores as follows: First, we calculated an average score separately for anxiety and depression per age. If a participant missed single items on a particular questionnaire ( $n=12$ ), we computed the average raw score from the non-missing items for this specific participant. Next, we standardized, for all participants, these average scores of anxiety and depression per age and averaged the anxiety and depression scores to combine them into one single score of internalizing symptoms per age (Cherlin, Chase-Lansdale, \& McRae, 1998; Haselager, Cillessen, van Lieshout, Riksen-Walraven, \& Hartup, 2002). If a participant missed a full questionnaire at a certain age $(n=25)^{2}$, the internalizing score for that specific participant at that specific age was based on the non-missing questionnaire. Higher scores indicated more self-reported internalizing symptoms. Some participants missed internalizing symptom scores at some of the four assessment points: $23 \%$ missed one, $13 \%$ missed two, and $4 \%$ missed three. The final self-reported internalizing scores were positively pairwise correlated across age ( $r$ s between 0.33 and 0.57 , all ps < 0.01), except between ages 9 and $14(r=0.22, p=0.061,95 \%$ $\mathrm{Cl}[-0.01,0.43])$, and between 9 and $17(r=0.14, p=0.224,95 \%$ $\mathrm{Cl}[-0.08,0.34])$.

\subsection{3 | Parent-reported internalizing symptoms}

For parent-reported internalizing symptoms we used the internalizing subscale of the Child Behavior Checklist (CBCL; Achenbach, 1991) at ages 5, 9, 12, 14, and 17, using age-appropriate versions (Table 1; Supporting Information Appendix S6). To account for different numbers of items in each version, we calculated an average overall score per age. Higher scores reflected more parent-reported internalizing symptoms. Similar to self-report 


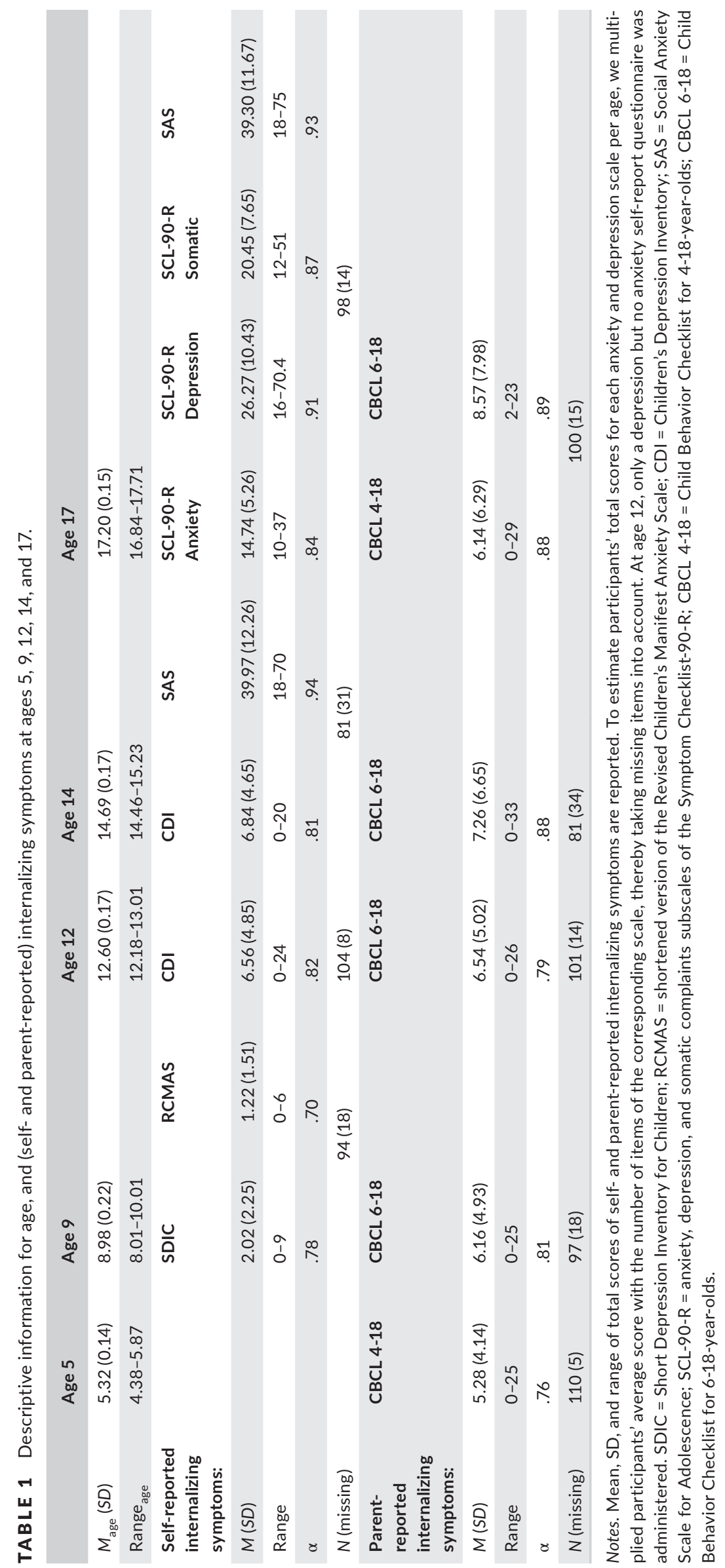


procedures, we determined an adjusted average score for participants who missed single items per age $(n=5)$. Contrary to selfreported procedures, standardization per age was not required, as the same questionnaire $(C B C L)$ with the same answer scale (0-2) was used across all ages. ${ }^{3}$ Parent-reported internalizing symptoms were positively pairwise correlated across age ( $r$ between 0.33 and 0.64 , all ps $<0.01$ ), except between ages 5 and $14(r=0.14, p=0.213,95 \% \mathrm{Cl}[-0.08,0.35])$, and between 5 and $17(r=0.20, p=0.052,95 \% \mathrm{Cl}[-0.002,0.38])$. Some participants missed the internalizing symptoms at some of the five assessment points: $23 \%$ missed one, $11 \%$ missed two, $5 \%$ missed three, and $3 \%$ missed four. Parent- and self-reported internalizing symptoms were correlated with each other at ages $9(r=0.31, p=0.005$, 95\% Cl [0.10, 0.49]), 14 ( $r=0.37, p=0.001,95 \% \mathrm{Cl}[0.17,0.55])$, and $17(r=0.34, p=0.001,95 \% \mathrm{Cl}[0.15,0.51])$, but not at age 12 $(r=0.00, p>0.250,95 \% \mathrm{Cl}[-0.20,0.19])$.

\subsection{4 | Parental stress}

Structured parent-child interactions were assessed for the quality of parental behavior to indicate parental stress at 15 months, 2.5, 5, 7, and 12 years of age (Niermann et al., 2015). Trained observers rated the age-appropriate parent-child interactions on five-sevenpoint scales (Erickson, Sroufe, \& Egeland, 1985): (a) supportive presence/provision of emotional support, (b) respect for the child's autonomy/non-intrusiveness, (c) effective structure/limit-setting, (d) quality of instructions, (e) hostility. We reversed the hostility scores, standardized each scale per age, and summed these scales to obtain one score of parental stress per age. Higher scores indicated lower parental stress. As these total scores were positively correlated between ages ( $r$ between 0.39 and $0.63, p<0.001$ ), they were averaged to one overall score of parental stress. Missing data (14\% missed one, $5 \%$ missed two, $3 \%$ missed three, $1 \%$ missed four measurement points) were handled by computing an average score from the non-missing observations.

\subsection{5 | Peer stress}

Social preference by peers was assessed at ages 9, 12, 13, and 16 to indicate peer stress (van den Berg, Burk, \& Cillessen, 2014). Using a well-established sociometric approach, participants and their classmates were asked to nominate classmates (excluding self-nominations) whom they liked most and whom they liked least. For each question, number of nominations received was counted and standardized within classrooms. A score for social preference was computed per age as the difference between the standardized most-liked and least-liked scores, and again standardized within classrooms. As these scores were positively correlated across age ( $r$ s between 0.26 and $0.63, p<0.05$ ), an average overall score of peer preference across age was calculated, with lower scores indicating being more disliked, so higher levels of peer stress. Good reliability and high stability of peer preference scores have been established previously (Jiang \& Cillessen, 2005). Missing values (25\% missed one, $14 \%$ missed two, $4 \%$ missed three measurement (a)

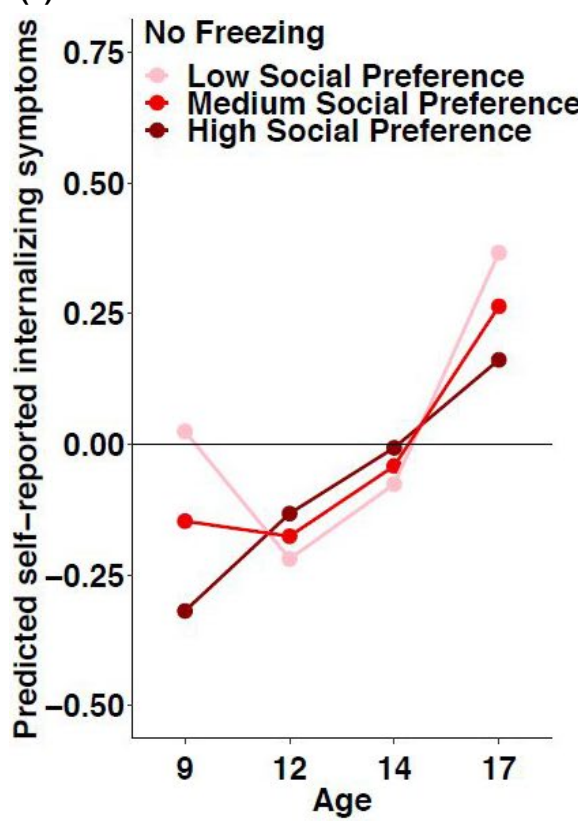

(b)

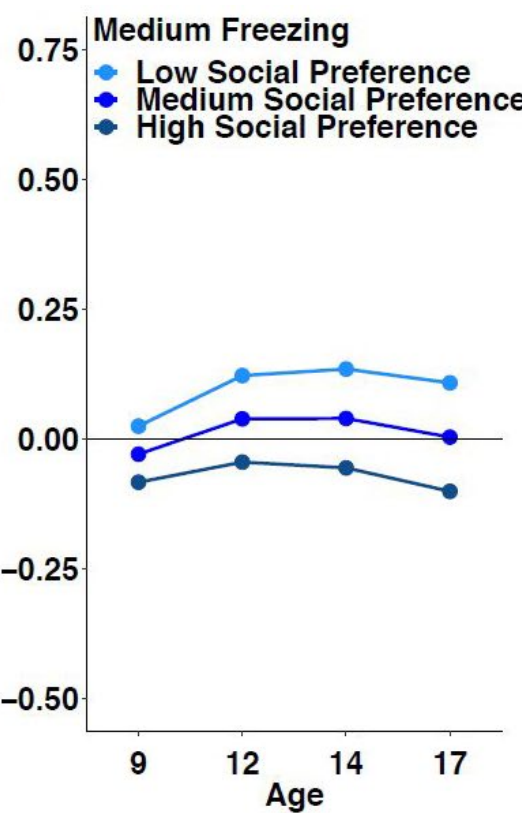

(c)

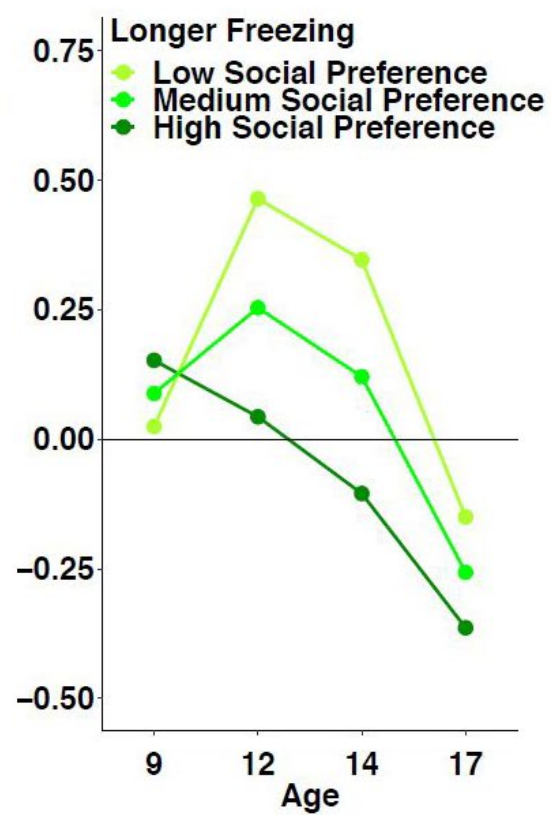

FIGURE 1 Model-based changes in self-reported internalizing symptoms at ages 9, 12, 14, and 17 as a function of infant freezing and peer preference (no freezing/low preference [1 SD below mean]; medium freezing/medium preference [ $M=0]$; longer freezing/high preference [1 SD above mean]). Whereas absent infant freezing was predictive of a continuous increase from relatively low to relatively high internalizing symptoms up until late adolescence (a), longer freezing was only related to relative increases in internalizing symptoms during early adolescence as a function of low peer preference, a pattern which decreased with age (c). Medium infant freezing was not predictive of alterations in internalizing symptoms (b). Standardized scores were used for self-reported internalizing symptoms 
points) were handled in a similar way as parental stress. Two participants were excluded from the social environment analyses for parent-reported and one for self-reported internalizing symptoms because they missed all peer preference scores.

\section{3 | Statistical analyses}

All analyses were conducted in R (version 3.3.2; R Core Team, 2016). To investigate the effect of infant freezing on changes in internalizing symptoms, we used a linear mixed-effects model approach (Imer function; Ime4 package; Bates, Maechler, Bolker, \& Walker, 2015). We conducted two linear mixed-effects models, including either self- or parent-reported internalizing symptoms as the dependent variable, and infant freezing as the independent variable. Also each model examined the polynomial (linear and quadratic) effects of age as a main effect and in interaction with infant freezing on internalizing symptoms. An advantage of linear mixed-effects models is that they can use all available data of the dependent variable of internalizing symptoms, even when some observations for some participants are missing. For detailed information on statistical analyses, see Supporting Information Appendix S7. To examine whether the experience of parental and/or peer stress moderated the association between infant freezing and relative changes in internalizing symptoms, we included parental and peer stress in the models each as main effect, as well as in two-way and three-way interactions with infant freezing and the linear and quadratic effects of age. Finally, we explored the role of 5-HTTLPR/rs25531 genotype in infant freezing. We used ranked-based regressions from the Rfit package (Kloke \& Mckean, 2012) to deal with the constrained distribution (between 0 and 1) of infant freezing.

\section{3 | RESULTS}

The mixed-effects model for self-reported internalizing symptoms resulted in interactions between linear age $\times$ infant freezing, $\chi^{2}(1)=7.41, p=0.007,95 \% \mathrm{Cl}[-0.04,-0.01]$ and quadratic age $\times$ infant freezing, $\chi^{2}(1)=8.38, p=0.007,95 \% \mathrm{Cl}[-0.04,-0.01]$. These interaction effects suggest that individuals' levels of infant freezing positively predicted increases in internalizing symptoms relative to others ${ }^{4}$ in the sample during early adolescence (Figure 1). However, during late adolescence, higher infant freezing predicted relatively decreasing levels of internalizing symptoms. More specifically, individuals showing longer infant freezing behavior (1 SD above mean) showed more internalizing symptoms relative to others at age 12 , but later showed relatively fewer internalizing symptoms (Figure 1c). In contrast, individuals who showed no ${ }^{5}$ infant freezing (1 SD below mean) displayed relatively fewer internalizing symptoms across adolescence before age 17. At age 17, they tended to report more symptoms than their peers (Figure 1a). Individuals with medium infant freezing $(M=0)$ showed no changes in their internalizing symptoms across age (Figure $1 \mathrm{~b}$; see Supporting Information Table S4 for results of the full model).
All reported effects remained when controlling for gender and externalizing symptoms (Supporting Information Appendices S9 and S10; Tables S4 and S6), and when predicting self-reported depressive and anxiety symptoms separately, except for the quadratic age $\times$ infant freezing interaction for self-reported anxiety symptoms. The absence of this latter effect likely results from the fact that the anxiety measurement at age 12 was missing (Supporting Information Appendix S11; Tables S7 and S8; Figure S2).

\section{1 | Infant freezing and social environment}

To test whether social environment moderated the effects of freezing on self-reported internalizing symptoms, we added parental and peer stress to the model. The results showed similar interactions for linear and quadratic age $\times$ infant freezing, $\chi^{2}(1)=9.12$, $p=0.006,95 \% \mathrm{Cl}[-0.04,-0.01]$ and $\chi^{2}(1)=10.11, p=0.002,95 \%$ $\mathrm{Cl}[-0.04,-0.01]$, respectively, replicating the effects just reported. Most critically, we observed a three-way interaction for peer preference $\times$ quadratic age $\times$ infant freezing, $\chi^{2}(1)=5.78, p=0.020$, $95 \% \mathrm{Cl}[0.003,0.03]$. This three-way interaction was interpreted based on Figure 1, illustrating model-based predictions. It suggests that only those youths who exhibited longer infant freezing behavior (1 SD above mean) and were disliked by their peers ( 1 SD below mean) displayed relatively higher internalizing symptoms at ages 12 and 14 (Figure 1c). In contrast, the above-reported relative increase in internalizing symptoms across adolescence in individuals with no infant freezing behavior (1 SD below mean) occurred irrespective of being liked or disliked by their peers (Figure 1a). Similarly, the absence of differences in internalizing symptoms across age in individuals with medium infant freezing $(M=0)$ remained, irrespective of being liked or disliked by their peers (Figure $1 \mathrm{~b}$ ). We observed no moderating effect for parental stress (parental stress $\times$ quadratic age $\times$ infant freezing), $\chi^{2}(1)=1.69, p=0.213,95 \% \mathrm{Cl}[-0.01,0.03]$. For results of the full model, see Supporting Information Table S4. As before, all reported effects remained when controlling for externalizing symptoms (Supporting Information Appendix S10; Table S6) and when predicting self-reported depressive and anxiety symptoms separately (Supporting Information Appendix S11; Tables S7 and S8).

Infant freezing did not predict changes in parent-reported internalizing symptoms and was not moderated by parental or peer stress (Supporting Information Appendix S8; Table S5).

\subsection{Serotonin transporter gene polymorphism}

We explored the role of genetic variation in serotonin signaling in deviant freezing, as seen in animals (Homberg, 2012). First, we explored whether 5-HTTLPR/rs25531 genotype ( $\mathrm{S}^{\prime}$-homozygotes vs $L^{\prime}$-carriers [ $L^{\prime} / L^{\prime}$ and $L^{\prime} / S^{\prime}$ combined]) could predict infant freezing in a linear fashion, which was not the case (estimate $=0.00, t=-0.04$, $p>0.250$ ). Then, we tested for an association with deviant freezing in both (extremely low and high) directions. We used centered freezing scores to compute participants' absolute score as the degree to which they deviated from the mean. Therefore, a higher 
score (more deviant freezing) reflected either absent or excessively long freezing. This model confirmed that $S^{\prime}$-homozygotes showed more deviant freezing behavior when compared with $L^{\prime}$-carriers (estimate $=-0.01, \quad t=-2.55, \quad p=0.012$; Supporting Information Figure S3). This result remained when controlling for gender (5-HTTLPR/rs25531: estimate $=-0.01, t=-2.47, p=0.015$; gender: estimate $=0.00, t=-0.42, p>0.250)$. However, we observed no main effect of 5 -HTTLPR/rs25531, when 5 -HTTLPR/rs25531 was coded as a predictor with three levels $\left(S^{\prime} / S^{\prime}, L^{\prime} / L^{\prime}, L^{\prime} / S^{\prime}\right.$-carriers; $F(2,92)=2.11, p=0.127)$. Finally, we checked whether genotype ( $S^{\prime}$-homozygotes vs L'-carriers) moderated the association between deviant freezing and participants' peak in self-reported internalizing symptoms. This was not the case. Nonetheless, the effect of deviant freezing on internalizing symptoms remained (Supporting Information Appendix S12).

\section{4 | DISCUSSION}

The aim of this study was to investigate whether early signs of alterations in freezing behavior predict changes in the relative development of internalizing symptoms. The results suggest that deviations in infant freezing-both longer and absent freezing-were associated with relative increases in self-reported internalizing symptoms at different ages during adolescence. Specifically, absent infant freezing predicted a relative increase in internalizing symptoms consistently across development, from relatively low symptom levels in childhood to relatively high levels in late adolescence. Longer infant freezing also predicted a relative increase in internalizing symptoms but only until early adolescence; this pattern was moderated by peer stress and was followed by a decrease afterwards. In light of the stability and conceptualization of freezing as a trait (Buss et al. (2004); Rogers et al. (2008); but see also Niermann et al. (2018)) as well as in light of the current observation of deviant freezing in $\mathrm{S}^{\prime}$ homozygotes of 5-HTTPLR/rs25531, our findings suggest that deviant infant freezing-both longer and absent freezing-may signal risk for the development of internalizing symptoms.

Previous work indicated that freezing in low-risk contexts predicts heightened stress responding, which is a potential risk for internalizing symptoms (Buss \& McDoniel, 2016). We extended these findings in three ways. Our results suggest that (a) threat-related freezing is also informative of developing internalizing symptoms, (b) not only increased but also absent freezing predicts internalizing symptoms, and (c) different types of deviant (longer vs absent) freezing predict different time-varying patterns of internalizing symptoms across age.

Our finding of increasing internalizing symptoms across development for individuals showing no infant freezing may at first seem surprising. It is, however, consistent with the notion that freezing is an adaptive response, facilitating perception, decision-making, and action preparation in threatening situations (Blanchard, 2017; Kozlowska et al., 2015; Roelofs, 2017). It is also in line with neurochemical theories suggesting that a decrease in inhibition in an aversive context may be a mechanism contributing to the development of affective disorders (Dayan \& Huys, 2009). This phenomenon of aversive disinhibition and excessive forms of inhibition, likewise, have been associated with altered serotonin signaling (due to depletion or genetic predisposition; Dayan \& Huys, 2009). In line with this work, our explorative analysis suggests that $S^{\prime}$-homozygotes of $5-H T T L P R / r s 25531$ show more deviant freezing than L'-carriers. This finding suggests a similar genetic basis of alterations in human freezing as previously reported for animals (Bethea et al., 2004; Homberg, 2012). However, 5-HTTLPR/rs25531 polymorphism did not moderate the association between freezing and internalizing symptoms in this study, suggesting that freezing-although related to $5-H T T L P R / r s 25531-$ is independently associated with internalizing symptoms. This is in line with the observation of inconsistent meta-analytic findings of 5-HTTLPR on internalizing symptoms (Bleys et al., 2018; Culverhouse et al., 2018).

Our finding of increasing internalizing symptoms in individuals with higher infant freezing is consistent with previous notions that reduced ability to recover from an initial freezing response is a potential signal of maladaptive stress coping. It may reduce an individual's flexibility to adequately respond to environmental changes during threat (Buss \& Larson, 2000; Hagenaars et al., 2014; Niermann et al., 2017). Additionally, it is consistent with findings linking prolonged freezing to increased internalizing symptoms (Kozlowska et al., 2015; Niermann et al., 2017).

Consistent with diathesis-stress models (e.g., Zuckerman, 1999), only individuals showing both longer infant freezing and high peer stress demonstrated a relative increase in self-reported internalizing symptoms, and did so only during early adolescence. Indeed, poor peer relationships have been related to internalizing disorders, whereas high quality of peer relationships may act as a buffer preventing the negative consequences of longer infant freezing (Deater-Deckard, 2001). Surprisingly, the association between longer infant freezing and relative increases in internalizing symptoms was only present during early adolescence, and reversed afterward. The latter observation contrasts with findings of increased risk for internalizing disorders in cross-sectional studies at various ages in inhibited children (for a meta-analysis, see Clauss \& Blackford, 2012). It is difficult, however, to compare these crosssectional findings directly with our longitudinal intrapersonal changes. Future studies testing these associations in an independent longitudinal sample are needed, which should also assess developmental changes in sensitivity to peer stimuli that may have affected our findings (Sebastian et al., 2010).

The quality of peer relationships moderated the association with internalizing symptoms only in individuals with longer but not with absent infant freezing. This effect could not be attributed to differences in quality of peer relationships (Supporting Information Appendix S13). It is possible that individuals showing no freezing-like behavior (and hence took less time to carefully assess their environment) were also less sensitive to the influence of peer stimuli. The fact that our moderation effect was only present for peer but not parental stress, is possibly due to adolescents' increased orientation 
toward their peers and less toward their parents (Sebastian et al., 2010).

Freezing has been described as a crucial component of behavioral inhibition, a trait describing stable individual differences in reactivity and regulation of fear that has been associated with risk for anxiety and depression (Clauss \& Blackford, 2012; Fox, Henderson, Marshall, Nichols, \& Ghera, 2005). The differential effects for reduced versus increased freezing in terms of temporal dynamics of internalizing symptom development suggest that it may be worth exploring freezing separately from other signs of behavioral inhibition. Notably, whereas behavioral inhibition typically consists of behaviors that can be related to both sympathetic and parasympathetic physiological stress responses (Fox et al., 2005), assessing freezing separately allows for a specific investigation of the parasympathetic state-a critical component in action selection and flexible stress coping (Kozlowska et al., 2015; Roelofs, 2017).

\section{1 | Interpretational issues}

To assess internalizing symptoms across development, we selected and standardized age-appropriate self-report questionnaires of anxiety and depression. The use of age-appropriate measures may have confounded the observed differences due to a change in measurement tool and should therefore be replicated in an independent sample. The alternative option, however,-selecting the same selfreport questionnaire for assessments from age 9 to late adolescence-would have had the disadvantage of not using age-optimized measures. Therefore, by selecting psychometrically sound, and ageappropriate measures (recommended by Cillessen \& Lansu, 2015; see also Supporting Information Appendix S5 for the justification, validity, and reliability of the measures), we feel we have optimized the validity and reliability of our measurements.

We did not observe any associations between individuals' infant freezing and parent-reported internalizing symptoms. However, adolescents are known to experience more internalizing symptoms than is typically recognized by parents, suggesting that they are the most reliable informants of their own emotions and thoughts (Sourander, Helstela, \& Helenius, 1999).

The increased internalizing symptoms observed in this study concern relative changes that did not exceed clinical cut-off scores (Supporting Information Appendix S11). However, even moderately increased levels of internalizing symptoms during adolescence have been linked to risk for later psychopathology (Reinherz, Paradis, Giaconia, Stashwick, \& Fitzmaurice, 2003). This suggests that the atypical freezing pattern observed in our study-reflecting either absent or increased freezing-may mark an individual's vulnerability to develop internalizing symptoms at some stage of their development. Using high-risk samples, future stress vulnerability and resilience studies should determine whether this is indeed the case.

During adolescence, a general increase in internalizing symptoms, particularly for girls, has been well-documented (Bongers, Koot, van der Ende, \& Verhulst, 2003). We also observed this increase in parent- and self-reported measures, suggesting that our sample was normative (Supporting Information Appendix S9; Figure S1).

\section{5 | CONCLUSIONS}

This prospective longitudinal study suggests that the absence of infant freezing behavior predicts a consistent pattern of relative increases in internalizing symptoms across adolescence, from relatively low symptom levels in childhood to relatively high levels in late adolescence. In contrast, longer infant freezing predicted relative increases in internalizing symptoms during early adolescence followed by a decrease during late adolescence. This effect was moderated by peer stress. These findings suggest that alterations in infant freezing-both longer and absent freezing-may mark individuals' vulnerability for the development of internalizing symptoms and may be an important marker for stress vulnerability and resilience studies.

\section{ACKNOWLEDGEMENTS}

The authors thank Marianne Riksen-Walraven, Sanny Smeekens, and Vivian Heuvelmans for their help in conceptualizing and rating infant freezing. The authors are grateful to Nicole Perry for her input, to all researchers who were involved in previous data assessments of the NLS, and particularly to the participants and their families for their ongoing participation.

\section{CONFLICTS OF INTEREST}

B. Franke has received educational speaking fees from Shire and Medice.

\section{DATA AVAILABILITY}

Data are available upon request from the Data Archiving and Networked Services (DANS): https://doi.org/10.17026/ dans-zpr-dejk.

\section{ENDNOTES}

${ }^{1}$ We also assessed freezing in a stranger situation (van Bakel \& RiksenWalraven, 2004). However, because only 34 of the 116 infants showed freezing episodes (one or more) in this situation, these data showed an uneven distribution, decreasing the likelihood of being able to link freezing to internalizing symptoms (see for results Supporting Information Appendix S4; Tables S2 and S3). Buss (2011) found that a robot confrontation elicited more fearful behavior compared to a stranger confrontation. This appears to be consistent with our observation of more freezing behavior in the robot condition than the stranger confrontation, suggesting that a robot might trigger stronger responses than a stranger in these experimental situations. Nevertheless, the freezing response coded for the stranger and robot conditions were correlated with each other $(r=0.21, p=0.003,95 \% \mathrm{Cl}[0.08,0.33]$; we used the rank based correlation coefficient Kendall's tau because of the constrained [i.e., between 0 and 1] distribution of infant freezing). 
${ }^{2}$ The results remained the same, when these participants $(n=25)$ who missed a full questionnaire at a certain age were removed from our analyses.

${ }^{3}$ Standardization of parent-reported internalizing symptoms per age resulted in similar results compared to unstandardized parent-reported internalizing symptoms. As expected, the main effect of linear age, $\chi^{2}(1)=0.05, p>0.250,95 \% \mathrm{Cl}[-0.02,0.02]$, was no longer significant because of the standardization.

${ }^{4}$ We used standardized scores for self-reported internalizing symptoms. Therefore, the effects reflect relative changes in internalizing symptoms (Cherlin et al., 1998; Haselager et al., 2002).

${ }^{5}$ Individuals scoring 1 SD below mean, represent all individuals who showed no infant freezing.

\section{ORCID}

Hannah C. M. Niermann iD https://orcid.org/0000-0002-9850-8771

Anna Tyborowska iD https://orcid.org/0000-0002-7621-3497

Barbara Franke iD https://orcid.org/0000-0003-4375-6572

\section{REFERENCES}

Achenbach, T.M. (1991). Manual for the child behavior checklist/4-18 and 1991 profile. Burlington, VT: Department of Psychiatry, University of Vermont.

Adenauer, H., Catani, C., Keil, J., Aichinger, H., \& Neuner, F. (2010). Is freezing an adaptive reaction to threat? Evidence from heart rate reactivity to emotional pictures in victims of war and torture. Psychophysiology, 47(2), 315-322. https://doi.org/10.1111/j.1469-8986.2009.00940.x

Bates, D., Maechler, M., Bolker, B., \& Walker, S. (2015). Fitting linear mixed-effects models using Ime4. Journal of Statistical Software, 67(1), 1-48. https://doi.org/10.18637/jss.v067.i01

Bethea, C.L., Streicher, J.M., Coleman, K., Pau, F.K.Y., Moessner, R., \& Cameron, J.L. (2004). Anxious behavior and fenfluramine-induced prolactin secretion in young rhesus macaques with different alleles of the serotonin reuptake transporter polymorphism (5HTTLPR). Behavior Genetics, 34, 295-307. https://doi.org/10.1023/ B:BEGE.0000017873.61607.be

Blanchard, D.C. (2017). Translating dynamic defense patterns from rodents to people. Neuroscience and Biobehavioral Reviews, 76, 22-28. https://doi.org/10.1016/j.neubiorev.2016.11.001

Bleys, D., Luyten, P., Soenens, B., \& Claes, S. (2018). Gene-environment interactions between stress and 5-HTTLPR in depression: A metaanalytic update. Journal of Affective Disorders, 226, 339-345. https:// doi.org/10.1016/j.jad.2017.09.050

Bongers, I.L., Koot, H.M., van der Ende, J., \& Verhulst, F.C. (2003). The normative development of child and adolescent problem behavior. Journal of Abnormal Psychology, 112(2), 179-192. https://doi. org/10.1037/0021-843X.112.2.179

Buss, K.A. (2011). Which fearful toddlers should we worry about? Context, fear regulation, and anxiety risk. Developmental Psychology, 47, 804-819. https://doi.org/10.1037/a0023227

Buss, K.A., Davidson, R.J., Kalin, N.H., \& Goldsmith, H.H. (2004). Context-specific freezing and associated physiological reactivity as a dysregulated fear response. Developmental Psychology, 40, 583-594. https://doi.org/10.1037/0012-1649.40.4.583

Buss, K.A., \& Larson, C.L. (2000). Adaptive and maladaptive fear-related behaviors: Implications for psychopathology from Kalin's primate model. In R.J. Davidson (Ed.), Anxiety, depression, and emotion (pp. 69-87). New York: Oxford University Press.

Buss, K.A., \& McDoniel, M. (2016). Improving the prediction of risk for anxiety development in temperamentally fearful children.
Current Directions in Psychological Science, 25(1), 14-20. https://doi. org/10.1177/0963721415611601

Campese, V., McCue, M., Lázaro-Muñoz, G., LeDoux, J.E., \& Cain, C.K. (2013). Development of an aversive Pavlovian-to-instrumental transfer task in rat. Frontiers in Behavioral Neuroscience, 7, 176. https://doi. org/10.3389/fnbeh.2013.00176

Cherlin, A.J., Chase-Lansdale, P.L., \& McRae, C. (1998). Effects of parental divorce on mental health throughout the life course. American Sociological Review, 63, 239-249. https://doi.org/10.2307/2657325

Cillessen, A.H., \& Lansu, T.A. (2015). Stability, correlates, and timecovarying associations of peer victimization from grade 4 to 12 . Journal of Clinical Child and Adolescent Psychology, 44(3), 456-470. https://doi.org/10.1080/15374416.2014.958841

Clauss, J.A., \& Blackford, J.U. (2012). Behavioral inhibition and risk for developing social anxiety disorder: A meta-analytic study. Journal of the American Academy of Child and Adolescent Psychiatry, 51(10), 1066-1075.e1061. https://doi.org/10.1016/j.jaac.2012.08.002

Craske, M.G. (2003). Anxious processes. In M.G. Craske (Ed.), Origins of phobias and anxiety disorders: Why more women than men? (pp. 71-98). Oxford: Elsevier. https://doi.org/10.1016/ B978-008044032-3/50005-8

Culverhouse, R.C., Saccone, N.L., Horton, A.C., Ma, Y., Anstey, K.J., Banaschewski, T., ... Bierut, L.J. (2018). Collaborative meta-analysis finds no evidence of a strong interaction between stress and 5-HTTLPR genotype contributing to the development of depression. Molecular Psychiatry, 23(1), 133-142. https://doi.org/10.1038/mp.2017.44

Dayan, P., \& Huys, Q.J. (2009). Serotonin in affective control. Annual Review of Neuroscience, 32, 95-126. https://doi.org/10.1146/annurev.neuro.051508.135607

Deater-Deckard, K. (2001). Annotation: Recent research examining the role of peer relationships in the development of psychopathology. Journal of Child Psychology and Psychiatry, 42(5), 565-579. https://doi. org/10.1017/S0021963001007272

Erickson, M.F., Sroufe, L.A., \& Egeland, B. (1985). The relationship between quality of attachment and behavior problems in preschool in a high-risk sample. Monographs of the Society for Research in Child Development, 50, 147-166. https://doi.org/10.2307/3333831

Fox, N.A., Henderson, H.A., Marshall, P.J., Nichols, K.E., \& Ghera, M.M. (2005). Behavioral inhibition: Linking biology and behavior within a developmental framework. Annual Review of Psychology, 56, 235262. https://doi.org/10.1146/Annurev.Psych. 55.090902.141532

Fragkaki, I., Roelofs, K., Stins, J., Jongedijk, R.A., \& Hagenaars, M.A. (2017). Reduced freezing in posttraumatic stress disorder patients while watching affective pictures. Frontiers in Psychiatry, 8, 39. https://doi.org/10.3389/fpsyt.2017.00039

Hagenaars, M.A., Oitzl, M., \& Roelofs, K. (2014). Updating freeze: Aligning animal and human research. Neuroscience and Biobehavioral Reviews, 47, 165-176. https://doi.org/10.1016/j.neubiorev.2014.07.021

Haselager, G.J., Cillessen, A.H., van Lieshout, C.F., Riksen-Walraven, J.M., \& Hartup, W.W. (2002). Heterogeneity among peer-rejected boys across middle childhood: Developmental pathways of social behavior. Developmental Psychology, 38(3), 446-456. https://doi. org/10.1037//0012-1649.38.3.446

Homberg, J.R. (2012). Serotonergic modulation of conditioned fear. Scientifica (Cairo), 2012, 821549. https://doi.org/10.6064/2012/821549

Jiang, X.L., \& Cillessen, A.H.N. (2005). Stability of continuous measures of sociometric status: A meta-analysis. Developmental Review, 25(1), 1-25. https://doi.org/10.1016/j.dr.2004.08.008

Kalin, N.H., \& Shelton, S.E. (1989). Defensive behaviors in infant rhesus monkeys: Environmental cues and neurochemical regulation. Science, 243, 1718-1721. https://doi.org/10.1126/science. 2564702

Kalin, N.H., \& Shelton, S.E. (2003). Nonhuman primate models to study anxiety, emotion regulation, and psychopathology. Annals of the New York Academy of Sciences, 1008, 189-200. https://doi.org/10.1196/ annals.1301.021 
Kloke, J.D., \& Mckean, J.W. (2012). Rfit: Rank-based estimation for linear models. The $R$ Journal, 4, 57-64.

Kozlowska, K., Walker, P., McLean, L., \& Carrive, P. (2015). Fear and the defense cascade: Clinical implications and management. Harvard Review of Psychiatry, 23(4), 263-287. https://doi.org/10.1097/ hrp.0000000000000065

Ly, V., Huys, Q.J., Stins, J.F., Roelofs, K., \& Cools, R. (2014). Individual differences in bodily freezing predict emotional biases in decision making. Frontiers in Behavioural Neurosciences, 8, 237. https://doi. org/10.3389/fnbeh.2014.00237

Ly, V., von Borries, A.K., Brazil, I.A., Bulten, B.H., Cools, R., \& Roelofs, K. (2016). Reduced transfer of affective value to instrumental behavior in violent offenders. Journal of Abnormal Psychology, 125, 657-663. https://doi.org/10.1037/abn0000166

Mullen, M., Snidman, N., \& Kagan, J. (1993). Free-play behavior in inhibited and uninhibited children. Infant Behavior and Development, 16 , 383-389. https://doi.org/10.1016/0163-6383(93)80043-8

Niermann, H.C.M., Figner, B., Tyborowska, A., Cillessen, A.H.N., \& Roelofs, K. (2018). Investigation of the stability of human freezinglike responses to social threat from mid to late adolescence. Frontiers in Behavioural Neurosciences, 12, 97. https://doi.org/10.3389/ fnbeh.2018.00097

Niermann, H.C.M., Figner, B., Tyborowska, A., van Peer, J., Cillessen, A.H.N., \& Roelofs, K. (2017). Defensive freezing responses link Hypothalamic-Pituitary-Adrenal-axis activity and internalizing symptoms in humans. Psychoneuroendocrinology, 82, 83-90. https://doi. org/10.1016/j.psyneuen.2017.05.001

Niermann, H.C.M., Ly, V., Smeekens, S., Figner, B., Riksen-Walraven, J.M., \& Roelofs, K. (2015). Infant attachment predicts bodily freezing in adolescence: Evidence from a prospective longitudinal study. Frontiers in Behavioral Neuroscience, 9, 263. https://doi.org/10.3389/ fnbeh.2015.00263

Qi, C., Roseboom, P.H., Nanda, S.A., Lane, J.C., Speers, J.M., \& Kalin, N.H. (2010). Anxiety-related behavioral inhibition in rats: A model to examine mechanisms underlying the risk to develop stress-related psychopathology. Genes, Brain, and Behavior, 9, 974-984. https://doi. org/10.1111/j.1601-183X.2010.00636.x

R Core Team. (2016). R: A language and environment for statistical computing. Vienna, Austria: R Foundation for Statistical Computing. Available from: https://www.R-project.org/.

Reinherz, H.Z., Paradis, A.D., Giaconia, R.M., Stashwick, C.K., \& Fitzmaurice, G. (2003). Childhood and adolescent predictors of major depression in the transition to adulthood. American Journal of Psychiatry, 160(12), 2141-2147. https://doi.org/10.1176/appi. ajp.160.12.2141

Roelofs, K. (2017). Freeze for action: Neurobiological mechanisms in animal and human freezing. Philosophical Transactions of the Royal Society of London. Series B, Biological Sciences, 372(1718), 1-10. https://doi. org/10.1098/rstb.2016.0206
Rogers, J., Shelton, S.E., Shelledy, W., Garcia, R., \& Kalin, N.H. (2008). Genetic influences on behavioral inhibition and anxiety in juvenile rhesus macaques. Genes, Brain and Behavior, 7, 463-469. https://doi. org/10.1111/j.1601-183X.2007.00381.x

Sebastian, C., Viding, E., Williams, K.D., \& Blakemore, S.J. (2010). Social brain development and the affective consequences of ostracism in adolescence. Brain and Cognition, 72(1), 134-145. https://doi. org/10.1016/j.bandc.2009.06.008

Sourander, A., Helstela, L., \& Helenius, H. (1999). Parent-adolescent agreement on emotional and behavioral problems. Social Psychiatry and Psychiatric Epidemiology, 34(12), 657-663. https://doi. org/10.1007/s001270050189

Stoffels, M., Nijs, M., Spinhoven, P., Mesbah, R., \& Hagenaars, M.A. (2017). Emotion avoidance and fear bradycardia in patients with borderline personality disorder and healthy controls. Journal of Behavior Therapy and Experimental Psychiatry, 57, 6-13. https://doi. org/10.1016/j.jbtep.2017.02.001

van Bakel, H.J.A., \& Riksen-Walraven, J.M. (2002). Parenting and development of one-year-olds: Links with parental, contextual, and child characteristics. Child Development, 73, 256-273. https://doi. org/10.1111/1467-8624.00404

van Bakel, H.J.A., \& Riksen-Walraven, J.M. (2004). Stress reactivity in 15-month-old infants: Links with infant temperament, cognitive competence, and attachment security. Developmental Psychobiology, 44, 157-167. https://doi.org/10.1002/dev.20001

van den Berg, Y.H.M., Burk, W.J., \& Cillessen, A.H.N. (2014). Identifying subtypes of peer status by preference and popularity: A cohort sequential approach. The Journal of Early Adolescence, 35, 1108-1137. https://doi.org/10.1177/0272431614554704

Zuckerman, M. (Ed.) (1999). Diathesis-stress models. In Vulnerability to psychopathology: A biosocial model (pp. 3-23). Washington, DC: American Psychological Association. https://doi.org/10.1037/10316-000

\section{SUPPORTING INFORMATION}

Additional supporting information may be found online in the Supporting Information section at the end of the article.

How to cite this article: Niermann HCM, Tyborowska A, Cillessen $\mathrm{AHN}$, et al. The relation between infant freezing and the development of internalizing symptoms in adolescence: A prospective longitudinal study. Dev Sci. 2019;22:e12763. https://doi.org/10.1111/desc.12763 